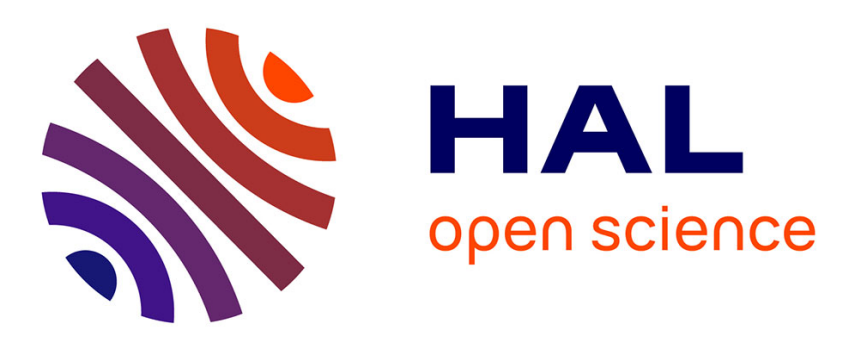

\title{
Three-dimensional terahertz computed tomography of human bones
}

Maryelle Bessou, Bruno Chassagne, Jean-Pascal Caumes, Christophe Pradère, Philippe Maire, Marc Tondusson, Emmanuel Abraham

\section{- To cite this version:}

Maryelle Bessou, Bruno Chassagne, Jean-Pascal Caumes, Christophe Pradère, Philippe Maire, et al.. Three-dimensional terahertz computed tomography of human bones. Applied optics, 2012, 51 (28), pp.6738-6744. 10.1364/AO.51.006738 . hal-00751516

\section{HAL Id: hal-00751516 https://hal.science/hal-00751516}

Submitted on 13 Nov 2012

HAL is a multi-disciplinary open access archive for the deposit and dissemination of scientific research documents, whether they are published or not. The documents may come from teaching and research institutions in France or abroad, or from public or private research centers.
L'archive ouverte pluridisciplinaire $\mathbf{H A L}$, est destinée au dépôt et à la diffusion de documents scientifiques de niveau recherche, publiés ou non, émanant des établissements d'enseignement et de recherche français ou étrangers, des laboratoires publics ou privés. 


\title{
Three-dimensional terahertz computed tomography of human bones
}

\author{
Maryelle Bessou, ${ }^{1}$ Bruno Chassagne,${ }^{2}$ Jean-Pascal Caumes, ${ }^{3}$ Christophe Pradère,${ }^{3}$ \\ Philippe Maire, ${ }^{4}$ Marc Tondusson, ${ }^{4}$ and Emmanuel Abraham ${ }^{4, *}$ \\ 'PACEA, Univ. Bordeaux, CNRS, UMR 5199, F-33405 Talence, France \\ ${ }^{2}$ ALPhANOV, Centre Technologique Optique et Lasers, F-33405 Talence, France \\ ${ }^{3}$ I2M-TREFLE, Univ. Bordeaux, ENSAM-CNRS, UMR 8508, F-33405 Talence, France \\ ${ }^{4}$ LOMA, Univ. Bordeaux, CNRS, UMR 5798, F-33405 Talence, France \\ *Corresponding author: em.abraham @loma.u-bordeaux1.fr
}

Received 24 July 2012; revised 23 August 2012; accepted 25 August 2012; posted 27 August 2012 (Doc. ID 173175); published 25 September 2012

\begin{abstract}
Three-dimensional terahertz computed tomography has been used to investigate dried human bones such as a lumbar vertebra, a coxal bone, and a skull, with a direct comparison with standard radiography. In spite of lower spatial resolution compared with x-ray, terahertz imaging clearly discerns a compact bone from a spongy one, with strong terahertz absorption as shown by additional terahertz time-domain transmission spectroscopy. (C) 2012 Optical Society of America

OCIS codes: $\quad 110.6795,110.6880,100.6950,110.7440,170.6510$.
\end{abstract}

\section{Introduction}

Radiography is considered as one of the most efficient techniques to analyze biological tissues such as human bones. It offers submillimeter spatial resolution and highly contrasted images depending on the radiodensity, the thickness, and the composition of the materials [1,2]. In the field of three-dimensional (3D) imaging, x-ray computed tomography (CT) is an ubiquitous technique, largely employed in medical imaging, which provides cross-sectional images of an object by analyzing the radiation transmitted by the sample through different incidence angles. However, the use of x-ray is sometimes not recommended regarding the ionizing power of the radiation and the difficulty of sample radiometric dating after $\mathrm{x}$-ray irradiation [3]. Radiography also requires strict safety rules based on the knowledge of the radiation effects and on the principles of protection. Only licensed users can manipulate $\mathrm{x}$-ray producing devices

$1559-128 \mathrm{X} / 12 / 286738-07 \$ 15.00 / 0$

(C) 2012 Optical Society of America and perform radiographic examination, respecting safe operating procedures and ensuring the wearing of personal dosimeters. Besides, an under-exploited domain of the electromagnetic spectrum, known as the terahertz $(\mathrm{THz})$ spectral region, has recently emerged as a possible powerful candidate for the analysis of opaque objects. Being nondestructive and contactless in nature, the $\mathrm{THz}$ wave can penetrate into nonconductive and nonpolar materials and offers complementary spectroscopic data for a better diagnosis and understanding of materials. $\mathrm{THz}$ imaging has been widely employed in the field of homeland security and more recently for the investigation of artwork related to cultural heritage in collaboration with curators from museums [4-7].

Surprisingly, up to now, very few studies have been conducted concerning the analysis of human bones by $\mathrm{THz}$ radiation. Mechanical properties of compact bone have been first investigated by $\mathrm{THz}$ timedomain transmission spectroscopy in the $0.1-$ $1.25 \mathrm{THz}$ spectral range [8]. No correlation was found between the values of the Young's modulus and the spectroscopic $\mathrm{THz}$ parameters. The authors 
concluded that $\mathrm{THz}$ spectroscopy cannot rival with dual energy x-ray absorptiometry (DEXA) to identify the bone mineral density. $\mathrm{THz}$ pulsed imaging has been used to measure the early symptoms of osteoarthritis [9]. In this study, $\mathrm{THz}$ reflection images of excised rabbit femoral condyles demonstrated that multiple reflections can provide quantitative information on cartilage substructure. Baughman et al. presented a spectral imaging of osseous tissues through time-domain transmission spectroscopy. However, their study was limited to identifying regions of different osseous tissue type within a thin cross section of the distal epiphysis from a chicken bone [10]. At last, Öhrström et al. compared x-ray and pulsed $\mathrm{THz}$ imaging in the case of an artificially embalmed ancient Egyptian human mummy hand and a macerated human lumbar vertebra [11]. They pointed out that conventional radiography naturally provided higher spatial resolution, whereas $\mathrm{THz}$ imaging was well-suited for the investigation of soft tissues such as cartilaginous structures.

None of these studies presented the alternative potential of $\mathrm{THz}$ radiation for $3 \mathrm{D}$ imaging using $\mathrm{CT}$. Since the first demonstration of $\mathrm{THz} \mathrm{CT}$ by Ferguson et al. in 2002 , few papers have been actually published since the technique suffers from strong limitations such as beam diffraction and severe Fresnel losses for high refractive index samples [12-18]. In this paper, after a characterization of the $\overline{\mathrm{THz}}$ spectroscopic properties of human bone, we present the first 3D $\mathrm{THz}$ CT analysis of selected dried human bones associated with a direct comparison with conventional radiography. This study is focused on dried human bones, owing to the strong absorption of $\mathrm{THz}$ radiation by water. Therefore, the main goal of our work is not directly addressed to medical applications but rather to archeology or biological anthropology.

\section{Experimental Methods}

\section{A. Materials}

Human bones consist of compact and spongy bones. Compact bone is more dense, smooth, and homogeneous, whereas spongy bone is an open network structure, with small and irregular adjacent cavities. As the sample hydration state is of primary importance for $\mathrm{THz}$ transmission, our study has been limited to dried samples. For spectroscopic studies, pellets of bone samples have been prepared from a dried human femur. The compact bone pellet is very thin $(0.265 \mathrm{~mm})$ with a thickness precision of $\pm 0.005 \mathrm{~mm}$ obtained with a digital calliper. Such a thin pellet was required in order to properly measure the amplitude of the $\mathrm{THz}$ transmitted wave, as explained in the following section. The spongy bone pellet is much thicker $(2 \mathrm{~mm})$ owing the fragile nature of the highly scattering internal structure. Consequently, the spongy bone pellet will highly scatter the incoming $\mathrm{THz}$ radiation.

For imaging, we selected various relevant human bones such as a lumbar vertebra, a skull, and a coxal bone. The human bones were obtained from osteological collections at de la Préhistoire à l'Actuel: Culture, Environnement et Anthropologie (PACEA). They were selected according to their morphology and their internal structure. In this current study, only anatomic features useful for the optimal understanding of the images are indicated. The vertebrae belong to the irregular bones. They consist of a body and a vertebral arch constituted with costal (laterally) and spinous processes (posteriorly). A lumbar vertebra was chosen because of its morphology, with a broader and thicker body compared with other vertebrae [Fig. 3(a)]. These morphometric features will allow us to optimize the reading of the images. The processes are thin and mainly made of compact bone, whereas the vertebral body is mostly constituted of cancellous bone enclosed with a thin layer of compact bone. The vault of skull and the coxal bones constitute flat bones. Thin, flattened, and usually a bit curved, they have two roughly parallel compact bone surfaces, with a layer of spongy bone between. For the skull, only the vault is composed with flat bones, whereas the face and base ones are classified as irregular bones. Numerous flat and thin bones with varied morphology compose the face, and their specific architecture creates several cavities (frontal sinuses, maxillary sinuses, nasal cavity, etc.). We will discuss in the Section 3 the influence of these cavities on $\mathrm{THz}$ imaging. In our study, the skull is interesting because of its important volume, the presence of the two types of bone tissues (spongy bone and compact bone), and the numerous adjoining empty spaces of the face. The current analyzed skull was formerly partially restored with plaster particularly both in the lateral and superior parts of parietal bones [Fig. 4(a)]. Finally, we propose to analyze a coxal bone that is a great flat bone [Fig. 5(a)]. The ilium wing does illustrate the specific feature of flat bones with spongy bone sandwiched between two thin layers of compact bone. This bone presents differences of thickness both in its superior (ilium) and inferior (pubis and ischium) parts. X-rays can distinguish very well these differences. We will see in the Section $\underline{3}$ how $\mathrm{THz}$ radiation can differentiate them.

\section{B. Methods}

$\mathrm{THz}$ time-domain transmission spectroscopy (TDS) was performed in order to measure the $\mathrm{THz}$ transmittance of human bone. To this aim, we used an amplified laser system $(800 \mathrm{~nm}, 1 \mathrm{kHz}, 1 \mathrm{~mJ}, 50 \mathrm{fs})$ to produce a $\mathrm{THz}$ beam consisting in a single-cycle $\mathrm{THz}$ wave generated by the interaction of the fundamental and the second-harmonic laser pulses in ionized air [19]. After transmission through the sample, this wave is measured by electro-optical sampling in a $100 \mu$ m-thick $\langle 110\rangle \mathrm{GaP}$ crystal in the $0.3-8 \mathrm{THz}$ spectral range, the lower frequency being limited by the $\mathrm{THz}$ emission within the plasma and the higher one by phonon absorption in the GaP crystal. This broadband spectrometer makes possible the evaluations of the frequency-dependent refractive 
index and absorption coefficient of the samples. The determination of the refractive index is important in order to quantify the deviation of the $\mathrm{THz}$ beam in the case of oblique incidence due to Fresnel laws, whereas the characterization of the absorption coefficient will indicate the global attenuation of $\mathrm{THz}$ radiation after propagating through bone samples.

The experimental setup of the continuous wave $\mathrm{THz}$ imaging system-shortly called the " $\mathrm{THz}$ scanner"-has already been described [20]. Briefly, it consists of a compact millimeter wave Gunn diode $(110 \mathrm{GHz}, 20 \mathrm{~mW})$ coupled with a horn antenna (Fig. 1). The output beam is focused on the sample, which is positioned on two-axes $X Y$ motorized stages. At the sample position, the beam diameter is diffraction limited and the final image is obtained point-by-point by raster scanning the sample in both horizontal and vertical directions. For the detection, we used a commercial low-cost pyroelectric sensor (Gentec EO) and an optical chopper connected to a lock-in amplifier. A two-dimensional (2D) transmission image of the sample is obtained by moving the object in the $X$ and $Y$ directions with a scan step of 1 or $2 \mathrm{~mm}$ in both directions. Owing to the low frequency of the emitting source, the spatial resolution of the $\mathrm{THz}$ scanner is limited to a few millimeters (110 $\mathrm{GHz}$ corresponds to a wavelength of $2.7 \mathrm{~mm}$ ). This is a strong limitation of the system for the analysis of human bones that require for imaging a spatial resolution better than $1 \mathrm{~mm}$. With a scan speed of 5 pixels /s, the acquisition time for a $(100 \times 100)$ pixels image size is about $30 \mathrm{~min}$. This portable $\mathrm{THz}$ scanner is well adapted for on-site imaging since its volume is about $(500 \mathrm{~mm} \times 500 \mathrm{~mm} \times 500 \mathrm{~mm})$ with a total weight less than $20 \mathrm{~kg}$. Moreover, as pointed out in the introduction, the imaging system is easy to install and completely safe for the sample and the operator, unlike radiography.

For 3D reconstruction, the sample is rotated in order to provide a different visualization of the object, such as in x-ray CT. From these tilted series, we are able to construct the sinogram of the object that represents, for a given horizontal slice, the evolution of the transmitted $\mathrm{THz}$ amplitude as a function of the rotation angle. Here, to get a reasonable acquisition time for 3D imaging, we selected a rotation step of $10^{\circ}$. In this case, we obtained the corresponding 18 projections in nearly $9 \mathrm{~h}$. In this procedure, it is important to position the rotation axis exactly in the middle of the 2D transmission images for any projection angle to avoid any future reconstruction artifacts due to lateral shifting. Moreover, as explained in our previous work, the $10^{\circ}$ rotation increment has been selected as a compromise between acquisition time and 3D imaging quality represented by spatial resolution, contrast, intensity, and geometric preservation [26]. For the final 3D reconstruction, we used the standard backprojection of the filtered projections (BFP) algorithm [21]. This reconstruction process is based on the inverse radon transform [22], which computes the final pixel values from the filtered projections. It is widely developed in $\mathrm{x}$-ray CT scan imaging and also used in $\mathrm{THz} \mathrm{CT}$. As will be shown in the following section, this standard algorithm is suitable for the final 3D reconstruction even if it is well known that it suffers from several disadvantages such as beam hardening, noise sensitivity, and geometric degradation in case of an insufficient number of projections. In a previous study, we tested alternative iterative reconstruction methods for THZ CT, such as the simultaneous algebraic reconstruction technique (SART) [23] or the ordered subsets expectation maximization (OSEM) method [24,25], with equivalent accuracy and imaging quality to BFP [26]. For a limited number of projections (typically less than 15), we emphasized a quantitative degradation of the BFP reconstruction, whereas the SART method can already offer optimized reconstruction quality. However, in this study, we selected the BFP algorithm for 3D reconstruction since it is extensively proposed in most of CT software tools.

For standard radiography, we used a medical x-ray tube including a rotating anode with a tungstenrhenium target on a molybdenum core. In order to properly visualize the structural characteristics of the human bones, three physical parameters were selected: the potential difference between the anode and the cathode of the $x$-ray tube corresponding to the penetrating power $(42-45 \mathrm{kV})$, the tube current (50 $\mathrm{mA})$, and the beam intensity related to the exposure time (25 mAs).

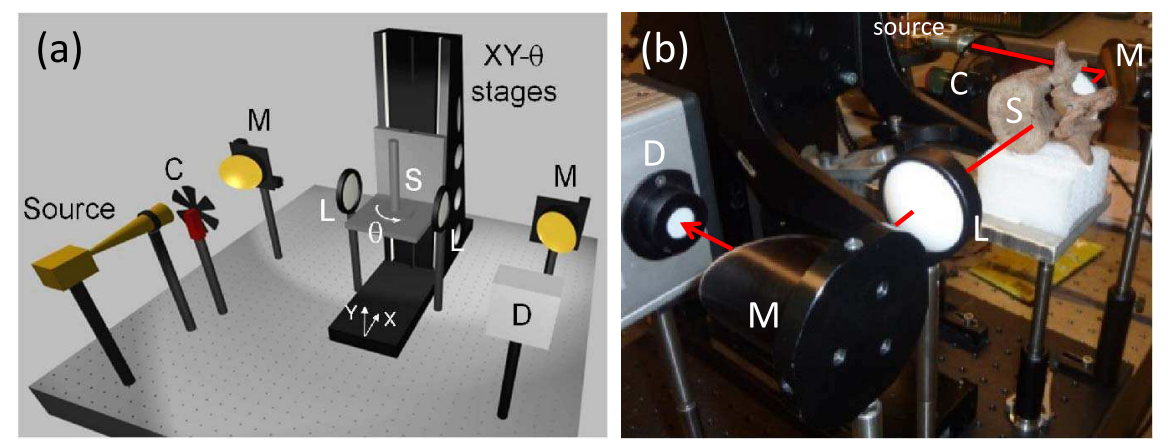

Fig. 1. (Color online) (a) Experimental setup. C, optical chopper; M, off-axis parabolic mirror $\left(f^{\prime}=150 \mathrm{~mm}\right) ; \mathrm{L}$, Teflon lens $\left(f^{\prime}=60 \mathrm{~mm}\right)$; $\mathrm{S}$, sample; D, pyroelectric detector. (b) Photograph of the THz scanner analyzing a human lumbar vertebra. 


\section{Results and Discussion}

\section{A. THz Time-Domain Transmission Spectroscopy}

At first, the spongy bone pellet was analyzed but the amplitude of the $\mathrm{THz}$ transmission was too weak compared to the noise level to quantitatively analyze this sample. The reason arises from the highly diffusive nature of this sample even if its thickness has been decreased as much as possible, as previously explained. For a quantitative study of this sample, a more precise analysis is in progress, taking into account the real scattering nature of the sample, according to the recent work of Joly et al. [27].

$\mathrm{THz}$ time-domain transmission spectroscopic properties of the compact bone pellet is presented in Fig. 2. In spite of the broadband time-domain spectrometer $(0.3-8 \mathrm{THz})$, the data are limited to the 0.3-2.75 $\mathrm{THz}$ spectral range owing to the strong absorption of $\mathrm{THz}$ radiation at higher frequencies. We observed that the refractive index slightly increases from 1.92 to 1.97 within the $0.3-1.9 \mathrm{THz}$ spectral range (blue curve). This measurement slightly differs from that of Stringer et al. (mean refractive index between 2.2 and 2.6, derived from the time-domain signal) [8]. One reason can be the problem of indirect comparison of biological tissues, which is always difficult owing to the inevitable presence of inhomogeneous structures within the samples. At higher frequencies, the amplitude of the refractive index fluctuates owing to poor signal-tonoise ratio. For $\mathrm{THz} \mathrm{CT}$, an important conclusion of this measurement is that the relatively high refractive index of compact bone will significantly deviate the direction of propagation of $\mathrm{THz}$ radiation in the case of oblique incidence onto the sample, according to the Fresnel laws. The absorption coefficient of compact bone also gradually increases from 10 to $420 \mathrm{~cm}^{-1}$ in the $0.3-2.75 \mathrm{THz}$ spectral range (red curve). This additional important result shows that $\mathrm{THz}$ imaging of human bone is almost impossible at frequencies higher than $1 \mathrm{THz}$ owing to a very high $\mathrm{THz}$ absorption, probably due to the presence of inorganic materials into the samples, such as

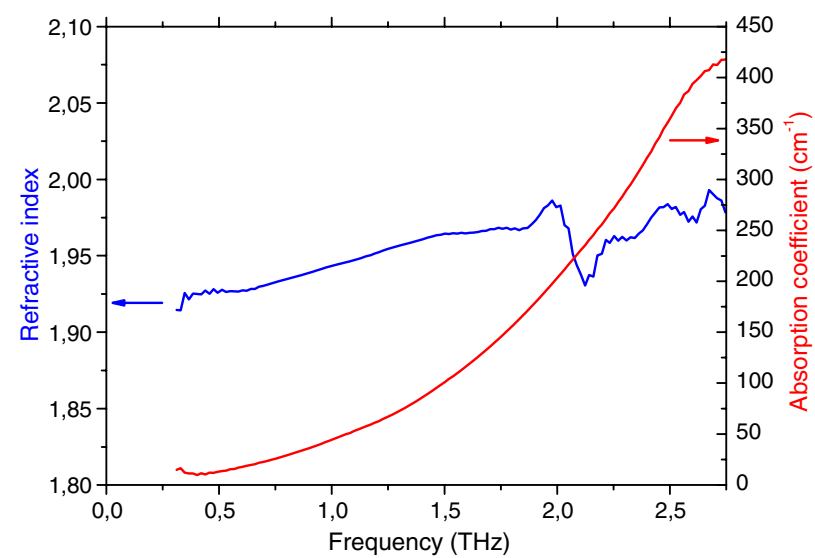

Fig. 2. (Color online) Refractive index (blue curve) and absorption coefficient (red curve) of compact bone. minerals (calcium) that represent the major components of the osseous tissues. To minimize the attenuation of $\mathrm{THz}$ radiation, it is more suitable to select a sub-THz frequency in spite of the degradation of imaging transverse resolution. The extrapolation of previous measurements provides an absorption coefficient less than $5 \mathrm{~cm}^{-1}$ at $110 \mathrm{GHz}$, corresponding to the emission frequency of the $\mathrm{THz}$ scanner. This estimation is in agreement with the direct evaluation of the absorption coefficient of compact bone $\alpha=(2.5 \pm$ $0.5) \mathrm{cm}^{-1}$ at this frequency obtained with use of the $110 \mathrm{GHz}$ source. Considering the signal-to-noise ratio of 10, 000:1 for the $\mathrm{THz}$ scanner, we can conclude that the total thickness of the compact bone should be roughly limited to $15 \mathrm{~mm}$ for effective imaging. As pointed out previously, besides the limited spatial resolution, this weak penetration depth constitutes another strong limitation of $\mathrm{THz}$ imaging compared to x-ray for the analysis of human bones.

\section{B. 2D THz Computed Tomography and Radiography}

Figure 3(a) shows the photograph of the dried human lumbar vertebra (superior view). The radiograph easily distinguishes with a submillimeter spatial resolution the two different types of bone structure, in particular the spongy bone microstructure [Fig. 3(b)]. As previously mentioned, the body of the vertebra is mainly composed with spongy bone, appearing in black on the radiograph and reflecting its low radiopacity. On the contrary, the limits of the body and the vertebral arch are made of more radiopaque compact bone. In spite of the reduced spatial resolution, $\mathrm{THz}$ imaging also reveals the density variations of the sample, indicating that compact bone exhibits higher $\mathrm{THz}$ absorption than the spongy one at $110 \mathrm{GHz}$ [Fig. 3(c)]. This additional observation was not possible by previous $\mathrm{THz}$ time-domain spectroscopy, as described above. We can also notice that the microspaces into the spongy bone do not disturb the propagation of the sub-THz wave [black area in the body of the vertebra, Fig. 3(c)].

Similarly, Fig. 4(a) shows the photograph of the dried human skull (right lateral view). The radiograph of the skull exhibits the limits of the cranial vault, the coronal suture indicated by the red arrow in the figure, the whole details of the face with the different cavities and the maxilla [Fig. 4(b)]. The dark zones on the radiograph arise from the empty spaces. THz imaging provides some similarities such as the important $\mathrm{THz}$ beam absorption by the vault bones and regions where the plaster is present (in the lateral and superior parts of parietal bones) [Fig. 4(c)]. The base and the face of the skull exhibit the same $\mathrm{THz}$ absorption except at the level of the orbital cavities. This can be explained by the multiple spaces in the base and the face of the skull that scatter the $\mathrm{THz}$ wave. Besides, in the case of a single cavity with smooth walls like those of the cranial vault, we clearly observed that the $\mathrm{THz}$ beam is not disturbed providing a higher $\mathrm{THz}$ transmission. Finally, the imaging of the coronal suture by both 


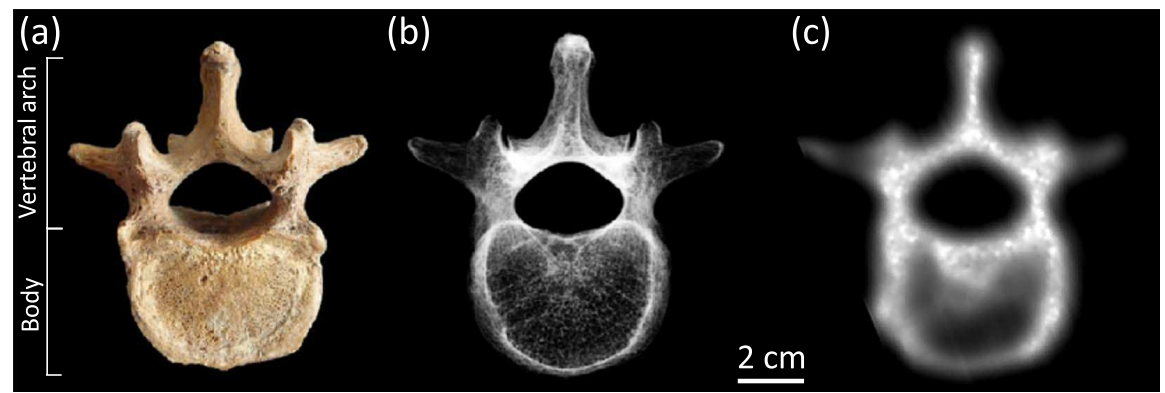

Fig. 3. (Color online) Human lumbar vertebra (superior view). (a) Photograph. (b) Radiograph. (c) THz imaging.

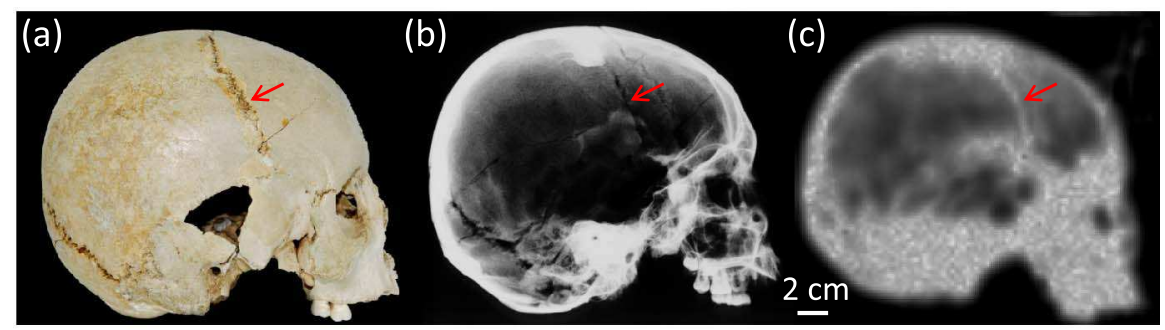

Fig. 4. (Color online) Human skull (right lateral view). (a) Photograph. (b) Radiograph. (c) THz imaging. The red arrows indicate the location of the coronal suture.

$\mathrm{THz}$ and x-ray calls our attention [indicated by the line pointed by the red arrow in Figs. 4(b) and 4(c)]. The coronal suture is formed by the union of two borders possessing serrated edges that articulate them together. Directly in the coronal suture region, the bone thickness is weaker and some little spaces can be present due to the possible postmortem fragmentation. This can explain the reason why on the radiograph the coronal suture appears darker than the rest of the cranial vault. However, in the $\mathrm{THz}$ image, the stronger $\mathrm{THz}$ absorption noted in this region may arise again from the scattering of the $\mathrm{THz}$ wave. In this example, a clear difference can thus be observed between x-ray and $\mathrm{THz}$ imaging.

\section{C. $3 \mathrm{D} \mathrm{THz} \mathrm{CT}$}

For 3D THz CT, we investigated the dried right coxal bone [photograph in Fig. 5(a)]. As noted previously, the coxal bone belongs both to flat bones (in the wing of ilium) and irregular bones (in the inferior part). On the radiograph, the wing of ilium is radiolucent, whereas the inferior part of the coxal bone is more radiopaque [Fig. 5(b)]. This result confirms the spongy structure of the wing of ilium and the higher proportion of compact bone in the inferior part associated with the higher thickness of the sample in this region. $\mathrm{THz}$ imaging properly illustrates all these characteristics with comparable color gradient [Fig. 5(c)]. Especially, in the wing of ilium where the $\mathrm{T} \overline{\mathrm{Hz}}$ absorption is reduced owing to its spongy nature, the x-ray and $\mathrm{THz}$ images look alike with the presence of well-defined absorption zones in the borders and in the lower part of the ilium wing. This additional example demonstrates the similarity of both methods for the imaging of spongy bone in spite of the lower spatial resolution of $\mathrm{THz}$ imaging.

For $3 \mathrm{D} \mathrm{THz}$ imaging, as previously explained, we selected a sample rotation step of $10^{\circ}$ from $0^{\circ}$ to $170^{\circ}$.

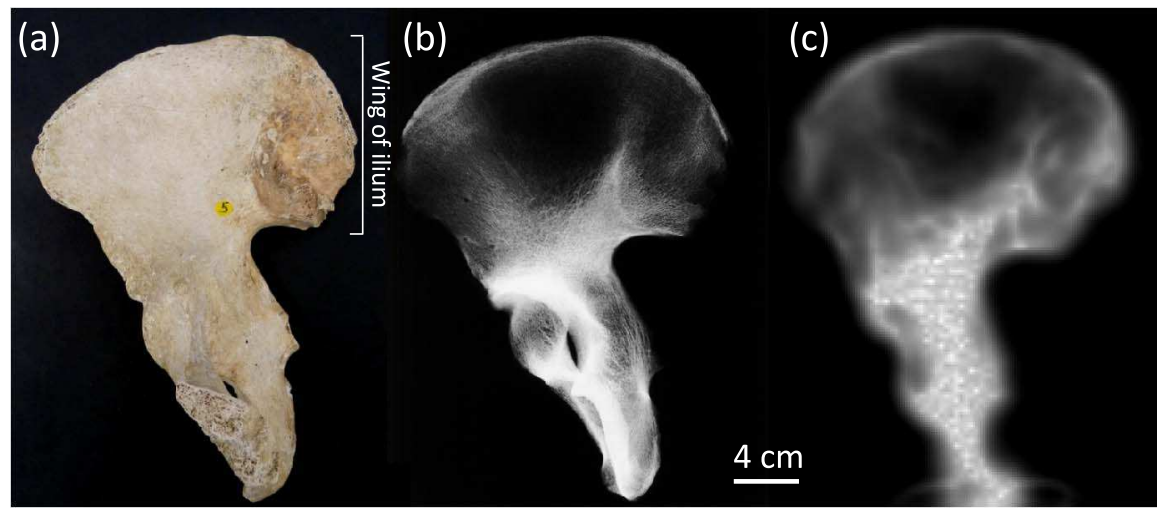

Fig. 5. (Color online) Right human coxal bone. (a) Photograph. (b) Radiograph. (c) THz imaging. 


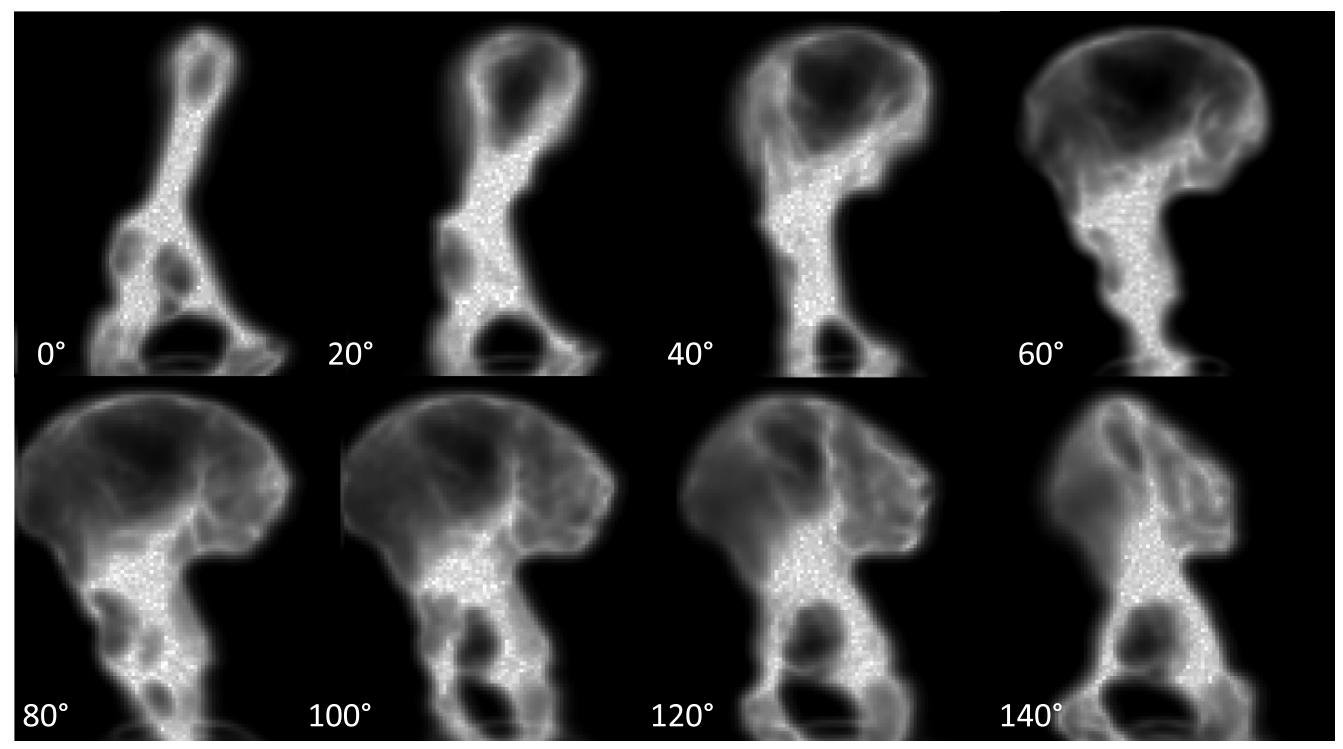

Fig. 6. THz imaging of right human coxal bone. Tilted series with a $20^{\circ}$ angular step. Whole tilted series available in "Media 1."

Figure 6 presents eight of the 18 available projections (angles from $0^{\circ}$ to $140^{\circ}$ with a $10^{\circ}$ rotation step). A whole visualization of the tilted series is available in Media 1. In spite of a poor spatial resolution, the projection $\mathrm{THz}$ images make it possible to differentiate compact and spongy bone into the sample, especially in the wing of the ilium. From these data, using the BFP algorithm, we can get a full 3D tomographic reconstruction of this coxal bone. Figure 7 (a) presents the $3 \mathrm{D}$ volumic reconstruction in order to qualitatively visualize the internal structures of the sample (also available in Media 2). Here, it is worth noting that the black hole appearing in the central part of the ilium wing comes from the relative transparency of the sample in this region. Another representation is given in Fig. 7(b), where the orthoslices with axial, coronal, and sagittal views make it possible to get some specific visualizations of the sample (also available in Media 3). This additional representation can be very useful on a practical point of view in order to analyze the images separately with a specific viewing angle.
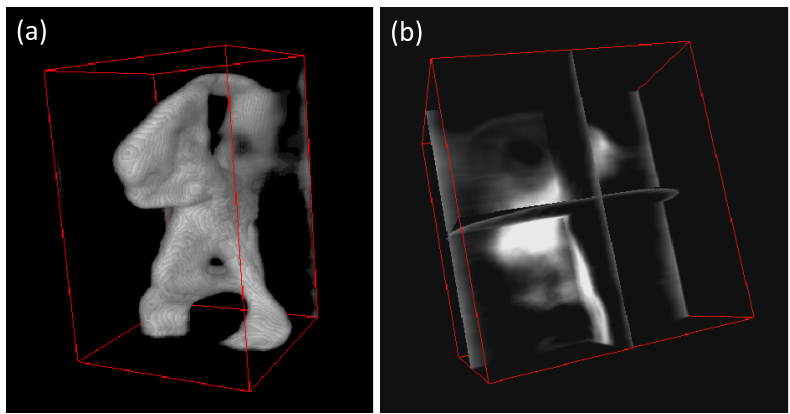

Fig. 7. (Color online) Right human coxal bone. (a) $3 \mathrm{D} \mathrm{THz}$ reconstruction (volume). Multimedia file in "Media 2". (b) 3D $\mathrm{THz}$ reconstruction (slices). From 3D tomographic reconstruction movie. Multimedia file in "Media 3." 3D tomographic reconstruction obtained with ImageJ software.

\section{Conclusion}

$2 \mathrm{D}$ and $3 \mathrm{D} \mathrm{THz}$ imaging have been compared to conventional radiography for the analysis of selected dried human bones. Radiography remains the standard imaging method for the analysis of the human skeleton with high penetration depth and submillimeter spatial resolution. Using $\mathrm{THz}$ time-domain transmission spectroscopy, we determined the refractive index and absorption coefficient of compact bone in the $0.3-2.75 \mathrm{THz}$ spectral range and concluded that $\mathrm{THz}$ imaging of bone tissues requires a sub-THz frequency owing to the high- $\mathrm{THz}$ absorption of bone material. Finally, using a Gunn diode at $110 \mathrm{GHz}$, we demonstrated that $\mathrm{THz}$ radiation can appear as an alternative and promising nonionizing electromagnetic wave to differentiate the two types of osseous tissues such as compact and spongy bones.

The authors gratefully acknowledge Bruno Maureille and Dominique Castex for the accessibility of the osteological collections from PACEA. They also thank the "Conseil Régional d'Aquitaine" and the CNRS for supporting the development of the Centre Optique et Laser d'Aquitaine (COLA) Platform at the LOMA.

\section{References}

1. O. Hahn, "X-ray fluorescence analysis on iron gall inks, pencils and coloured crayons," Stud. Conserv. 50, 23-32 (2005).

2. M. P. Morigi, F. Casali, M. Bettuzzi, R. Brancaccio, and V. d'Errico, "Application of x-ray computed tomography to cultural heritage diagnostics," Appl. Phys. A 100, 653-661 (2010).

3. M. J. Aitken, Thermoluminescence Dating (Academic, 1985).

4. J. B. Jackson, J. Bowen, G. Walker, J. Labaune, G. Mourou, M. Menu, and K. Fukunaga, "A survey of terahertz applications in cultural heritage conservation science," IEEE Trans. Terahertz Sci. Technol. 1, 220-231 (2011).

5. J.-P. Caumes, A. Younus, S. Salort, B. Chassagne, B. Recur, A. Ziéglé, A. Dautant, and E. Abraham, "Terahertz tomographic imaging of XVIIIth dynasty Egyptian sealed pottery," Appl. Opt. 50, 3604-3608 (2011). 
6. K. Fukunaga, E. Cortes, A. Cosentino, I. Stünkel, M. Leona, I. N. Duling III, and D. T. Mininberg, "Investigating the use of terahertz pulsed time domain reflection imaging for the study of fabric layers of an Egyptian mummy," J. Eur. Opt. Soc. Rapid Commun. 6, 11040 (2011).

7. M. Bessou, H. Duday, J.-P. Caumes, S. Salort, B. Chassagne, A. Dautant, A. Ziéglé, and E. Abraham, "Advantage of terahertz radiation versus x-ray to detect hidden organic materials in sealed vessels," Opt. Commun. 285, 4175-4179 (2012).

8. M. R. Stringer, D. N. Lund, A. P. Foulds, A. Duddin, E. Berry, R. E. Miles, and A. G. Davies, "The analysis of human cortical bone by terahertz time-domain spectroscopy," Phys. Med. Biol. 50, 3211-3219 (2005).

9. W. C. Kan, W. S. Lee, W. H. Cheung, V. P. Wallace, and E. Pickwell-MacPherson, "Terahertz pulsed imaging of knee cartilage," Biomed. Opt. Express 1, 967-974 (2010).

10. W. Baughman, D. S. Wilbert, S. Balci, M. Bolus, P. Kung, and S. M. Kim, "Application of terahertz spectral imaging for the identification of osseous tissue," in Proceedings of 36th International Conference on Infrared, Millimeter and Terahertz Waves (IRMMW-THz) (IEEE, 2011), pp. 1-2.

11. L. Öhrström, A. Bitzer, M. Walther, and F. J. Rühli, "Technical note: terahertz imaging of ancient mummies and bone," Am. J. Phys. Anthropol. 142, 497-500 (2010).

12. B. Ferguson, S. Wang, D. Gray, D. Abbot, and X. C. Zhang, "T-ray computed tomography," Opt. Lett. 27, 1312-1314 (2002).

13. S. Wang, B. Ferguson, D. Abbott, and X. C. Zhang, "T-ray imaging and tomography," J. Biol. Phys. 29, 247-256 (2003).

14. S. Wang and X. C. Zhang, "Pulsed terahertz tomography," J. Phys. D 37, R1-R36 (2004).

15. M. M. Awad and R. A. Cheville, "Transmission terahertz waveguide-based imaging below the diffraction limit," Appl. Phys. Lett. 86, 221107 (2005).

16. X. Yin, B. W. H. Ng, B. Ferguson, and D. Abbott, "Wavelet based local tomographic image using terahertz techniques," Digit. Signal Process. 19, 750-763 (2009).
17. A. Brahm, M. Kunz, S. Riehemann, G. Notni, and A. Tünnermann, "Volumetric spectral analysis of materials using terahertz tomography techniques," Appl. Phys. B 100, 151-158 (2010)

18. E. Abraham, A. Younus, C. Aguerre, P. Desbarats, and P. Mounaix, "Refraction losses in terahertz computed tomography," Opt. Commun. 283, 2050-2055 (2010).

19. D. J. Cook and R. M. Hochstrasser, "Intense terahertz pulses by four-wave rectification in air," Opt. Lett. 25, 1210-1212 (2000).

20. A. Younus, S. Salort, B. Recur, P. Desbarats, P. Mounaix, J.-P. Caumes, and E. Abraham, "Millimeter wave tomographic scanner for large size opaque object inspection with different refractive index contrasts," Proc. SPIE 7837, 783709 (2010).

21. G. T. Herman, Image Reconstruction From Projections: The Fundamentals of Computerized Tomography (Academic, 1980).

22. J. Radon, "Uber die Bestimmung von Funktionen durch ihre Integralwerte langs gewisser Mannigfaltigkeiten," Ber. Ver. Sachs. Akad. Wiss. Leipzig, Math-Phys. Kl 69, 262 (1917).

23. A. H. Andersen and A. C. Kak, "Simultaneous algebraic reconstruction technique (SART): a superior implementation of the ART algorithm," Ultrason. Imag. 6, 81-94 (1984).

24. L. A. Shepp and Y. Vardi, "Maximum likelihood reconstruction for emission tomography," IEEE Trans. Med. Imaging 1, 113-122 (1982).

25. H. M. Hudson and R. S. Larkin, "Accelerated image reconstruction using ordered subsets of projection data," IEEE Trans. Med. Imaging 13, 601-609 (1994).

26. B. Recur, A. Younus, S. Salort, P. Mounaix, B. Chassagne, P. Desbarats, J.-P. Caumes, and E. Abraham, "Investigation on reconstruction methods applied to 3D terahertz computed tomography," Opt. Express 19, 5105-5117 (2011).

27. S. Joly, F. Garet, and J.-L. Coutaz, "Accurate determination of the complex refractive index of scattering materials by $\mathrm{THz}$ time-domain spectroscopy," presented at the 3rd EOS Topical Meeting on Terahertz Science and Technology (TST 2012), Prague, Czech Republic, 17-20 June 2012. 